\title{
A CONTRIBUTION TO THE THEORY OF ASYMPTOTIC MARTINGALES
}

\author{
by ALLAN GUT
}

(Received 22 February, 1981)

1. Introduction. During the last few years several articles on asymptotic martingales (amarts) have appeared. The first unified treatment was given by Edgar and Sucheston in [7], where further references can be found. The purpose of this paper is to add some further results to the theory of amarts.

An important result is that an adapted sequence with an integrable supremum (together with a suitable sequence of $\sigma$-algebras) is an amart if and only if it is almost surely convergent, see [1, Corollary 1] and [7, Proposition 2.2]. This result is slightly sharpened in $\$ 4$ and as an application an amart related to the Marcinkiewicz law of large numbers is obtained.

Stability problems, i.e. the question of when a function of an amart is an amart, have been studied by Bellow (see [3], [4]). Some further results in this area are presented in $\$ 5$. The proofs make frequent use of the theorem of $\$ 4$.

Finally, in $\$ 6$, a Riesz decomposition theorem is given for the descending case. Contrary to the ascending case (see [7, Theorem 3.2]) uniqueness does not necessarily hold.

I wish to thank Peter Sjögren for valuable discussions.

2. Notations and definitions. We follow the notation of [7]. Let $(\Omega, \mathscr{F}, P)$ be a probability space, put $N=\{1,2, \ldots\},-N=\{\ldots,-2,-1\}$ and let $D$ denote either $N$ or $-N$. Further, let $\left\{\mathscr{F}_{n}\right\}_{n \in D}$ be an increasing family of sub- $\sigma$-algebras of $\mathscr{F}_{\mathcal{F}}$, i.e. $\mathscr{F}_{n} \subset \mathscr{F}_{m}$ if $n \leq m$, and set $\mathscr{F}_{\infty}=\sigma\left\{\bigcup_{n \in N} \mathscr{F}_{n}\right\}$ and $\mathscr{F}_{-\infty}=\bigcap_{n \in-N} \mathscr{F}_{n}$. The set of all bounded stopping times is denoted by $T\left(T_{D}, T_{N}, T_{-N}\right)$. With the definition $\tau \leq \sigma \Leftrightarrow \tau(\omega) \leq \sigma(\omega)$ for almost all $\omega \in \Omega, T_{N}$ is filtering to the right and $T_{-N}$ is filtering to the left.

Definrrion 2.1. The net $\left(a_{\tau}\right)_{\tau \in T}$ of real numbers converges to $a$ if and only if for all $\varepsilon>0$ there is $\tau_{0} \in T_{D}$ such that for all $\tau \in T_{N}$ with $\tau \geq \tau_{0}$ (for all $\tau \in T_{-N}$ with $\tau \leq \tau_{0}$ ) we have $\left|a_{\tau}-a\right|<\varepsilon$.

REMARK 2.2. An equivalent definition, which is frequently used, is that the sequence $\left\{a_{\tau_{n}}\right\}_{n \in N}$ converges for every increasing (decreasing) sequence, $\left\{\tau_{n}\right\}_{n \in N}, \tau_{n} \in T$ (see [2], [7], and also $[14$, p. 96]).

A sequence $\left\{X_{n}\right\}_{n \in D}$ is adapted if $X_{n}$ is $\mathscr{F}_{n}$-measurable for all $n \in D$. For $\tau \in T$ the random variable $X_{\tau}$ is defined by the relation $\left(X_{\tau}\right)(\omega)=X_{\tau(\omega)}(\omega)$. If the sequence of $\sigma$-algebras, $\left\{\mathscr{F}_{n}\right\}_{n \in D}$, is not specified it is assumed that $\mathscr{F}_{n}=\sigma\left\{X_{k} ; k \leq n\right\}$ for all $n \in D$.

Glasgow Math. J. 23 (1982) 177-186. 
DEFINITION 2.3. Let $\left\{X_{n}\right\}_{n \in D}$ be an integrable family of random variables which is adapted to $\left\{\mathscr{F}_{n}\right\}_{n \in D}$. We call $\left\{X_{n}, \mathscr{F}_{n}\right\}_{n \in D}$

(i) a martingale if $E X_{\tau}$ is constant for all $\tau \in T$,

(ii) an asymptotic martingale (amart) if the net $\left(E X_{\tau}\right)_{\tau \in T}$ is convergent,

(iii) a semiamart if the net $\left(E X_{\tau}\right)_{\tau \in T}$ is bounded.

Remark 2.4. By choosing $\tau_{1} \equiv n$ and $\tau_{2}$ equals $m$ on $A \in \mathscr{F}_{m}$ and equals $n$ on $A^{c}$, where $m \leq n$, it is easy to verify that a sequence satisfying $(i)$ necessarily satisfies the defining relation of a martingale. The converse follows from the optional sampling theorem.

REMARK 2.5. Every martingale is trivially an amart and, by [7, Lemma 1.2], every amart is a semiamart.

3. Some examples. This section contains some examples which will be of use later. Throughout, $(\Omega, \mathscr{F}, P)$ is the Lebesgue measure space on $[0,1]$.

EXAMPLE 3.1. For $p \geq 1$, let

$$
X_{2 n}^{(p)} \equiv 0 \quad \text { and } \quad X_{2 n+1}^{(p)}(\omega)=\left\{\begin{array}{lll}
2^{n / p} & \text { if } & \omega \in\left(0,2^{-n}\right) \\
0 & \text { if } & \omega \in\left[2^{-n}, 1\right)
\end{array}\right.
$$

for $n=1,2, \ldots$

For $p=1,\left\{X_{n}^{(1)}, \mathscr{F}_{n}^{(1)}\right\}_{n \in N}$ is an a.s. convergent semiamart that fails to be an amart, see $[\mathbf{1}$, p. 19].

If $p>1$, then $E \sup _{n}\left|X_{n}\right|=\sum_{k=1}^{\infty} 2^{n / p} \cdot 2^{-(n+1)}<\infty$. Since the sequence is a.s. convergent, an application of [7, Proposition 2.2], shows that $\left\{X_{n}^{(p)}, \mathscr{F}_{n}^{(p)}\right\}_{n \in N}$ is an amart.

EXAMPLE 3.2. This example is related to that of Sudderth [15, p. 2145]. Let $p>1 / 2$ and define

$$
Y_{i}^{n}(\omega)= \begin{cases}n^{1 / p} & \text { if } \omega \in\left[(i-1) / n^{2}, i / n^{2}\right) \\ 0 & \text { otherwise }\end{cases}
$$

for $i=1,2, \ldots, n$ and $n=1,2, \ldots$.

The sequence $\left\{X_{n}^{(p)}\right\}_{n \in N}$ is now defined as the sequence $Y_{1}^{1}, Y_{1}^{2}, Y_{2}^{2}, \ldots, Y_{1}^{n}$, $Y_{2}^{n}, \ldots, Y_{n}^{n}, \ldots$. It is easily verified that

$$
X_{n}^{(p)} \rightarrow 0 \text { a.s. and in } L^{1} \quad \text { as } n \rightarrow \infty .
$$

Define $\left\{\tau_{n}\right\}_{n \in N}$ as follows:

$$
\tau_{n}(\omega)=\left\{\begin{array}{l}
\frac{(n-1) n}{2}+\min \left\{k \leq n ; Y_{k}^{n}(\omega) \neq 0\right\} \\
\frac{n(n+1)}{2} \text { if } Y_{k}^{n}(\omega)=0 \text { for } 1 \leq k \leq n
\end{array}\right.
$$


Thus, $\tau_{n}$ equals the index of the $X$ which corresponds to the first $Y$ among $Y_{1}^{n}, \ldots, Y_{n}^{n}$ that is non-zero and $\tau_{n}$ equals the index of the $X$ that corresponds to the last $Y$ among $Y_{1}^{n}, \ldots, Y_{n}^{n}$ if the latter are all zero. Clearly, $\tau_{n} \in T$ and $\tau_{n}>\infty$ as $n \rightarrow \infty$. Furthermore,

$$
E X_{\tau_{n}}^{(\mathrm{p})}=E \max \left\{Y_{1}^{n}, \ldots, Y_{n}^{n}\right\}=n^{(1 / p)-1} .
$$

Let $1 / 2<p<1$. Then $E X_{\tau_{n}}^{(p)} \rightarrow+\infty$ as $n \rightarrow \infty$, which together with (3.1) yields an example of a uniformly integrable a.s. convergent sequence which fails to be a semiamart.

Next, let $p=1$. Then $E X_{\tau_{n}}^{(1)}=1$ and in view of (3.1) it follows that $\left\{X_{n}^{(1)}, \mathscr{F}_{n}^{(1)}\right\}_{n \in N}$ fails to be an amart. Further, since $X_{\tau_{n}}^{(1)} \rightarrow 0$ a.s. as $n \rightarrow \infty$, it follows that $\left\{X_{\tau_{n}}^{(1)}\right\}_{n=1}^{\infty}$, and hence that $\left\{X_{\tau}^{(1)}\right\}_{\tau \in T}$, is not uniformly integrable.

Finally, let $p>1$. Then, since $P\left(\sup _{n}\left|X_{n}^{(p)}\right|=k^{1 / p}\right)=\frac{1}{k(k+1)}$ it follows that $E \sup \left|X_{n}^{(p)}\right|<\infty$, which together with (3.1) and [7, Proposition 2.2] shows that $\left\{X_{n}^{(p)}, \mathscr{F}_{n}^{(p)}\right\}_{n \in N}$ is an amart.

EXAmple 3.3. Let $p \geq 1$ and define

for $n=1,2, \ldots$.

$$
X_{-n}^{(p)}(\omega)=\left\{\begin{array}{lll}
2^{n / p} & \text { if } & \omega \in\left(0,2^{-n}\right) \\
0 & \text { if } & \omega \in\left[2^{-n}, 1\right)
\end{array}\right.
$$

We first note that

$$
X_{n}^{(p)} \rightarrow 0 \quad \text { a.s. as } n \rightarrow \infty \text { and that }\left\{X_{n}^{(p)}\right\}_{n \in-N} \text { is } L^{1} \text {-bounded. }
$$

Let $p=1$ and introduce the (finite) stopping time $\tau$ by

$$
\tau(\omega)=\left\{\begin{array}{c}
\inf \left\{k \in-N ; X_{k}^{(p)}(\omega) \neq 0\right\} \\
-1 \text { if all } X_{k}^{(p)}(\omega)=0
\end{array}\right.
$$

and the sequence $\left\{\tau_{n}\right\}_{n \in N}, \tau_{n} \in T_{-N}$ by $\tau_{n}=\tau \vee(-n)$. A simple computation yields

$$
E X_{\tau_{n}}^{(1)}=\frac{n+1}{2} \rightarrow \infty \text { as } n \rightarrow \infty,
$$

which shows that $\left\{X_{n}^{(1)}, \mathscr{F}_{n}^{(1)}\right\}_{n \in-N}$ cannot be a semiamart.

If $p>1$, then $E \sup \left|X_{n}^{(p)}\right|<\infty$ and so, by (3.3) and [7, Proposition 2.2], it follows that $\left\{X_{n}^{(p)}, \mathscr{F}_{n}^{(p)}\right\}_{n \in-N}$ is an amart.

Remark 3.4. Note that if $D=N$ and $X_{n}$ is defined as $X_{-n}^{(1)}$ of Example 3.3 and $\mathscr{F}_{n}=\sigma\left\{X_{k} ; 1 \leq k \leq n\right\}$, then $\left\{X_{n}, \mathscr{F}_{n}\right\}_{n \in N}$ is an a.s. convergent martingale which is not uniformly integrable, see Doob [6, pp. 347-348]. The different behaviours for $D=N$ and $D=-N$ seem to be due to the fact that it is possible to stop $\left\{X_{n}^{(1)}\right\}_{n \in D}$ at $\sup X_{n}^{(1)}$ (which is not integrable) with a finite stopping time if $D=-N$ (i.e. $\tau$ in Example 3.3), something that is not possible when $D=N$. 
EXample 3.5. Let $p>1 / 2$, define $\left\{Y_{i}^{n} ; 1 \leq i \leq n, n \geq 1\right\}$ as in Example 3.2, define $\left\{X_{n}^{(p)}\right\}_{n \in-N}$ as $\ldots, Y_{n}^{n}, \ldots, Y_{2}^{n}, Y_{1}^{n}, \ldots, Y_{2}^{2}, Y_{1}^{2}, Y_{1}^{1}$ and let $\mathscr{F}_{-n}=\sigma\left\{X_{k} ; k \leq-n\right\}$.

First, note that

$$
X_{n}^{(p)} \rightarrow 0 \quad \text { a.s. and in } \quad L^{1} \quad \text { as } n \rightarrow-\infty .
$$

To continue the analogy with Example 3.2 define $\left\{\tau_{n}\right\}_{n \in N}$ by letting $\tau_{n}$ equal the index of the $X$ which corresponds to the last $Y$ among $Y_{1}^{n}, \ldots, Y_{n}^{n}$ that is non-zero and let $\tau_{n}$ equal the index of the $X$ that corresponds to the first $Y$ among $Y_{1}^{n}, \ldots, Y_{n}^{n}$ if the latter are all zero. Thus $\tau_{n} \in T_{-N}, \tau_{n} \downarrow-\infty$ and

$$
E X_{\tau_{n}}^{(p)}=n^{(1 / p)-1} .
$$

Just as above the case $1 / 2<p<1$ yields an example of a uniformly integrable a.s. convergent sequence which fails to be a semiamart, when $p=1\left\{X_{n}^{(1)}\right\}_{n \in-N}$ is uniformly integrable, while $\left\{X_{\tau}^{(1)}\right\}_{\tau \in T}$ is not and for $p>1\left\{X_{n}^{(p)}, \mathscr{F}_{n}^{(p)}\right\}_{n \in-N}$ is an amart.

4. A convergence theorem. The following result strengthens Proposition 2.2 of [7], (for $D=N$ see also [1, Corollary 1]).

THEOREM 4.1. Let $\left\{X_{n}\right\}_{n \in D}$ be adapted to $\left\{\mathscr{F}_{n}\right\}_{n \in D}$ and such that $\left\{X_{r}\right\}_{\tau \in T}$ is uniformly integrable. The following assertions are equivalent:

(i) $X_{n}$ converges a.e.

(ii) $\left\{X_{n}, \mathscr{F}_{n}\right\}_{n \in D}$ is an amart.

For a related result for the case $D=N$, continuous time and finite stopping times, see [12, T 11. Corollaire].

Since the above uniform integrability condition is satisfied for every semiamart if $D=-N$ (see [7, Theorem 2.9]) the following corollary is immediate.

COROLlaRY 4.2. Let $\left\{X_{n}, \mathscr{F}_{n}\right\}_{n \in-N}$ be an a.s. convergent semiamart. Then $\left\{X_{n}, \mathscr{F}_{n}\right\}_{n \in-N}$ is an amart.

Remark 4.3. There exist a.s. convergent semiamarts that are not amarts if $D=N$. See $[\mathbf{1}$, p. 19] (Example 3.1 above with $p=1$ ).

RemarK 4.4. Since $\left|X_{\tau}\right| \leq \sup \left|X_{n}\right|$ for all $\tau \in T$ it is clear that $E \sup \left|X_{n}\right|<\infty$ implies that $\left\{X_{\tau}\right\}_{\tau \in T}$ is uniformly integrable. Further, according to Blackwell and Dubins [5, Theorem 2], there are a.s. convergent martingales (and hence amarts), $\left\{X_{n}, \mathscr{F}_{n}\right\}_{n \in D}$, which are uniformly integrable, and thus such that $\left\{X_{\tau}\right\}_{\tau \in T}$ is uniformly integrable (see Meyer $\left[13\right.$, p. 126]), for which $E \sup \left|X_{n}\right|$ is not finite. For $D=-N$ see also Remark 4.7 below.

Remark 4.5. The examples of Section 3 show that Theorem 4.1 and Corollary 4.2 cannot be (essentially) improved.

Proof of Theorem 4.1 (i) $\Rightarrow$ (ii). The case $D=N$. Let $\left\{\tau_{n}\right\}_{n \in N}$, be an increasing sequence of bounded stopping times. Since $X_{n} \rightarrow X$, say, a.s. as $n \rightarrow \infty$ it follows that 
$X_{\tau_{n}} \rightarrow X$ a.s. as $n \rightarrow \infty$ and since, in particular, $\left\{X_{\tau_{n}}\right\}_{n=1}^{\infty}$ is uniformly integrable we conclude that $E X_{\tau_{n}} \rightarrow E X$ as $n \rightarrow \infty$, which in view of $[14$, p. 96] (see also Remark 2.2 above) implies that $\left\{X_{n}, \mathscr{F}_{n}\right\}_{n \in N}$ is an amart.

The proof for the case $D=-N$ is the same.

(ii) $\Rightarrow$ (i) The uniform integrability implies in particular that $\left\{X_{n}\right\}_{n \in D}$ is $L^{1}$-bounded and thus a.s. convergent (see e.g. [7, Theorem 2.3]).

As an application an amart related to the Marcinkiewicz strong law of large numbers (see [11, pp. 242-243]) is presented. Recall that the sequence of arithmetic means constitutes a reversed martingale in the case of the classical Kolmogorov strong law of large numbers.

EXAMPLE 4.6. Let $\left\{\xi_{n}\right\}_{n=1}^{\infty}$ be independent, identically distributed (i.i.d.) random variables. Suppose that $E\left|\xi_{1}\right| r<\infty$ for $0<r<2$ and further that $E \xi_{1}=0$ if $1<r<2$. Put

$$
X_{-n}=\left\{\begin{array}{lll}
n^{-1 / r} \cdot \sum_{k=1}^{n} \xi_{k} & \text { if } & 1 \leq r<2 \\
n^{-1} \cdot\left|\sum_{k=1}^{n} \xi_{k}\right|^{r} & \text { if } & 0<r<1
\end{array}\right.
$$

and $\mathscr{F}_{-n}=\sigma\left\{X_{k} ; k \leq-n\right\}$, for $n=1,2, \ldots$ Then $\left\{X_{n}, \mathscr{F}_{n}\right\}_{n \in-N}$ is an amart.

Proof. Let $r=1$. Since $\left\{X_{n}, \mathscr{F}_{n}\right\}_{n \in-N}$ is a martingale and since martingales are amarts we are done.

Let $1<r<2$. Then $E \sup \left|X_{n}\right|<\infty$ by [9, p. 904]. The Marcinkiewicz law implies that $X_{n} \rightarrow 0$ a.s. as $n \rightarrow-\infty$ and so an application of [7, Proposition 2.2] yields the desired conclusion.

Now, let $0<r<1$. Set $Y_{-n}=n^{-1} \cdot \sum_{k=1}^{n}\left|\xi_{k}\right|^{r}$ and $Z_{-n}=E\left(\left|\xi_{1}\right|^{r} \mid \mathscr{F}_{-n}\right)$, where $n=$ $1,2, \ldots$.

Since $X_{-n}=n^{-1} \cdot\left|\sum_{k=1}^{n} \xi_{k}\right|^{r} \leq n^{-1} \cdot \sum_{k=1}^{n}\left|\xi_{k}\right|^{r}=Y_{-n}$ and since $\left\{X_{n}\right\}_{n \in-N}$ is adapted it follows (recalling interchangeability) that

Thus,

$$
X_{-n}=E^{\mathscr{F}_{-n}} X_{-n} \leq E^{\mathscr{F}_{-n}} Y_{-n}=Z_{-n}
$$

$$
X_{n} \leq Z_{n} \text { for } n=\ldots,-3,-2,-1 \text {. }
$$

Now, $\left\{Z_{n}, \mathscr{F}_{n}\right\}_{n \in-N}$ is a martingale and thus uniformly integrable. It therefore follows from martingale theory that $\left\{Z_{\tau}\right\}_{\tau \in T}$ is uniformly integrable (see [13, p. 126]). Finally, (4.1) implies that $\left\{X_{\tau}\right\}_{\tau \in T}$ is uniformly integrable from which the amart property follows by Theorem 4.1.

Remark 4.7. In Example 4.6 we used [7, Proposition 2.2] for the case $1<r<2$. This was possible because $E \sup _{n}\left|n^{-1 / r} \cdot \sum_{k=1}^{n} \xi_{k}\right|<\infty$. For the case $0<r<1$ the same proposition is not applicable because $E \sup n^{-1} \cdot\left|\sum_{k=1}^{n} \xi_{k}\right|^{r}<\infty$ if and only if $E\left|\xi_{1}\right|^{r} \cdot \log ^{+}\left|\xi_{1}\right|<\infty$ (see [8, 
Theorem 3.2]) and the latter condition was not assumed. We thus have obtained an example (for $D=-N$ ), where Theorem 4.1 applies and where [7, Proposition 2.2] does not.

5. Stability. This section deals with the following problem: Given an amart $\left\{X_{n}, \mathscr{F}_{n}\right\}_{n \in D}$ and a function $\varphi: R \rightarrow R$, when is $\left\{\varphi\left(X_{n}\right), \mathscr{F}_{n}\right\}_{n \in D}$ an amart?

The first result of this kind is that the conclusion holds for $\varphi(x)=|x|, x^{+}(=\max \{0, x\})$ and $x^{-}(=-\min \{0, x\})$, provided $\left\{X_{n}\right\}_{n \in D}$ is $L^{1}$-bounded when $D=N$, see [1, Lemma 2] for $D=N$ and [7, Corollary 1.4]. For the case $D=N$, Bellow [3], [4] gives necessary and sufficient conditions on $\varphi$ for the conclusion to hold.

The present paper also covers the case $D=-N$. We also investigate which further assumptions must be made on the amart for the conclusion to remain valid when the necessary conditions on $\varphi$ no longer are satisfied.

Following Bellow [3], [4] such problems are called stability problems.

THEOREM 5.1. Let $\left\{X_{n}, \mathscr{F}_{n}\right\}_{n \in D}$ be an amart. If $D=N$, assume in addition that $\left\{X_{n}\right\}_{n \in N}$ is $L^{\prime}$-bounded. Let $\varphi: R \rightarrow R$ be a function such that

(i) $\varphi$ is continuous and

(ii) $\lim _{x \rightarrow \infty} \frac{\varphi(x)}{x}$ and $\lim _{x \rightarrow-\infty} \frac{\varphi(x)}{x}$ exist and are finite.

Then, $\left\{\varphi\left(X_{n}\right), \mathscr{F}_{n}\right\}_{n \in D}$ is an $L^{1}$-bounded amart.

Remark 5.2. The cases $\varphi(x)=|x|, x^{+}$and $x^{-}$mentioned above are obviously included. For $D=N$, Bellow [4, Theorem 2] shows that (i) and (ii) are necessary and sufficient for $\left\{\varphi\left(X_{n}\right), \mathscr{F}_{n}\right\}_{n \in N}$ to be an $L^{1}$-bounded amart.

Proof. The proof differs from that given in [4] in that it makes use of Theorem 4.1 and Corollary 4.2. However, just as in [4] it suffices to prove the theorem for the case $X_{n} \geq 0, \varphi(0)=0$ and $\lim _{x \rightarrow \infty} \frac{\varphi(x)}{x}=0$.

By the amart convergence theorem ([7, Theorem 2.3]) we know that $X_{n}$ converges a.s. as $n \rightarrow \infty(n \rightarrow-\infty)$ and thus, by (i), also that

$$
\varphi\left(X_{n}\right) \text { converges a.s. as } n \rightarrow \infty(n \rightarrow-\infty)
$$

By invoking the results of Section 4 it therefore remains to show that

$$
\begin{gathered}
\left\{\varphi\left(X_{n}\right), \mathscr{F}_{n}\right\}_{n \in-N} \text { is a semiamart for the case } D=-N, \text { and } \\
\left\{\varphi\left(X_{\tau}\right)\right\}_{\tau \in T} \text { is uniformly integrable for the case } D=N .
\end{gathered}
$$

We first consider the $L^{1}$-boundedness of $\left\{\varphi\left(X_{\tau}\right)\right\}_{\tau \in T}$. By assumption, $x^{-1} \cdot|\varphi(x)|<\varepsilon$ if $x>M$ and $|\varphi(x)| \leq \varphi_{0}$, say, if $0 \leq x \leq M$. Thus,

$$
\begin{aligned}
E\left|\varphi\left(X_{\tau}\right)\right| & =E\left|\varphi\left(X_{\tau}\right)\right| . I\left\{X_{\tau} \leq M\right\}+E\left|\varphi\left(X_{\tau}\right)\right| . I\left\{X_{\tau}>M\right\} \\
& \leq \varphi_{0} . P\left(X_{\tau} \leq M\right)+\varepsilon E X_{\tau} . I\left\{X_{\tau}>M\right\} \leq \varphi_{0}+\varepsilon \sup _{\tau} E X_{\tau}<\infty,
\end{aligned}
$$


since amarts are semiamarts. Thus, $\left\{\varphi\left(X_{n}\right), \mathscr{F}_{n}\right\}_{n \in D}$ is a semiamart and, in particular, if $D=-N$ the proof is complete.

Now, let $D=N$. A similar argument together with the maximal lemma (see e.g. [7, Lemma 1.1]) yields

$$
\begin{aligned}
& E\left|\varphi\left(X_{\tau}\right)\right| . I\left\{\left|\varphi\left(X_{\tau}\right)\right|>A\right\} \\
&=E\left|\varphi\left(X_{\tau}\right)\right| . I\left\{\left|\varphi\left(X_{\tau}\right)\right|>A, X_{\tau} \leq M\right\}+E\left|\varphi\left(X_{\tau}\right)\right| . I\left\{\left|\varphi\left(X_{\tau}\right)\right|>A, X_{\tau}>M\right\} \\
& \leq \varphi_{0} . P\left(\left|\varphi\left(X_{\tau}\right)\right|>A\right)+\varepsilon E X_{\tau} \leq \varphi_{0} \cdot A^{-1} \cdot \sup _{\tau} E\left|\varphi\left(X_{\tau}\right)\right|+\varepsilon \sup _{\tau} E X_{\tau}<\infty .
\end{aligned}
$$

Consequently,

$$
\limsup _{A \rightarrow \infty} E\left|\varphi\left(X_{\tau}\right)\right| . I\left\{\left|\varphi\left(X_{\tau}\right)\right|>A\right\} \leq \varepsilon \sup _{\tau} E X_{\tau}
$$

and (5.3) follows since $\varepsilon$ may be chosen arbitrarily small. The proof is terminated.

Now, suppose that $\varphi: R \rightarrow R$ is a function for which Theorem 5.1 ( $i$ ) does not hold. As pointed out in $\left[4\right.$, p. 286] one can always find a sequence of real numbers $\left\{a_{n}\right\}$, which is amart and such that $\left\{\varphi\left(a_{n}\right)\right\}$ is not. We therefore turn to the problem of finding what additional assumptions on the amart are needed (together with $(i)$ ) for the conclusion of Theorem 5.1 to remain valid when (ii) no longer holds.

THEOREM 5.3. Let $\left\{X_{n}, \mathscr{F}_{n}\right\}_{n \in D}$ be an amart and $\varphi: R \rightarrow R$ a continuous function such that $\lim _{x \rightarrow+\infty} \frac{\varphi(x)}{x}$ and $\lim _{x \rightarrow-\infty} \frac{\varphi(x)}{x}$ do not exist (finitely).

(a) If $D=N$ assume in addition that $\left\{X_{n}\right\}_{n \in N}$ is $L^{1}$-bounded and that $\left\{\varphi\left(X_{\tau}\right)\right\}_{\tau \in T}$ is uniformly integrable. Then $\left\{\varphi\left(X_{n}\right), \mathscr{F}_{n}\right\}_{n \in N}$ is an $L^{1}$-bounded amart.

(b) If $D=-N$ assume further that $\left\{\varphi\left(X_{n}\right), \mathscr{F}_{n}\right\}_{n \in-N}$ is a semiamart. Then $\left\{\varphi\left(X_{n}\right), \mathscr{F}_{n}\right\}_{n \in-N}$ is an amart.

Proof. The amart convergence theorem and the continuity of $\varphi$ together imply that $\varphi\left(X_{n}\right)$ converges a.s. as $n \rightarrow \infty(n \rightarrow-\infty)$. The amart property now follows immediately from Theorem 4.1 and Corollary 4.2 .

Remark 5.4. After reduction to the case $X_{n} \geq 0, \varphi(0)=0$ and $\lim _{x \rightarrow+\infty} x^{-1} \cdot \varphi(x)=0$ the proof of Theorem 5.1 consisted of showing the validity of (5.2) and (5.3) above. In the present theorem the corresponding properties are supposed to hold. However, following these remarks, some examples are presented to show that the theorem is (essentially) the best possible.

REMARK $5.5 . D=N$. It is easily seen by an estimate related to those used to show (5.2) and (5.3) that the assumption that $\left\{X_{n}\right\}_{n \in N}$ is $L^{1}$-bounded can be dropped if $\alpha_{1}=\liminf _{x \rightarrow+\infty}\left|x^{-1} \cdot \varphi(x)\right|$ and $\alpha_{2}=\liminf _{x \rightarrow-\infty}\left|x^{-1} \cdot \varphi(x)\right|$ both are positive, because the $L^{1}-$ 
boundedness then follows from the uniform integrability of $\left\{\varphi\left(X_{\tau}\right)\right\}_{\tau \in T}$. However, if $\alpha_{1}=\alpha_{2}=0$ this cannot be done as is seen by the following example.

Let $\left\{\xi_{n}\right\}_{n \in N}$ be a sequence of i.i.d. random variables such that $P\left(\xi_{n}=\pi\right)=$ $P\left(\xi_{n}=-\pi\right)=1 / 2$. Put $X_{n}=\sum_{k=1}^{n} \xi_{k} \quad$ and $\mathscr{F}_{n}=\sigma\left\{X_{k} ; k \leq n\right\}, \quad n=1,2, \ldots$ Then $\left\{X_{n}, \mathscr{F}_{n}\right\}_{n \in N}$ is a martingale (and hence an amart), $E\left|X_{n}\right| \sim \sqrt{ }(2 n)$ as $n \rightarrow \infty$, i.e. $\left\{X_{n}\right\}_{n \in N}$ is not $L^{1}$-bounded. Now, choose $\varphi(x)=|x \cdot \sin x|$, (for which $\alpha_{1}=\alpha_{2}=0$ and $\limsup _{|x| \rightarrow \infty}\left|x^{-1} \cdot \varphi(x)\right|=1$ ). Clearly $\varphi\left(X_{n}\right) \equiv 0$ for all $n$, in particular, $\left\{\varphi\left(X_{\tau}\right)\right\}_{\tau \in T}$ is uniformly integrable.

REMARK 5.6. If one of the limits $\lim _{x \rightarrow-\infty} x^{-1} \cdot \varphi(x)$ and $\lim _{x \rightarrow+\infty} x^{-1} \cdot \varphi(x)$ exists, finite and the other does not, then, by considering the positive and negative parts separately (cf. [4]) the assumptions on $\left\{\varphi\left(X_{\tau}\right)\right\}_{\tau \in T}$ and $\left\{\varphi\left(X_{n}\right)\right\}_{n \in-N}$, for $D=N$ and $D=-N$, respectively, can be reduced to assumptions on one part only, by applying Theorem 5.1 to the other part. Similarly, if for example $\alpha_{1}>0$ and $\alpha_{2}=0$, where $\alpha_{1}$ and $\alpha_{2}$ are defined as in Remark 5.5. As an example, consider

$$
\varphi(x)=\left\{\begin{array}{lll}
x^{2} & \text { if } & x \geq 0 \\
\sqrt{ }|x| & \text { if } & x<0
\end{array}\right.
$$

Then, for $D=N$, if $\left\{X_{n}, \mathscr{F}_{n}\right\}_{n \in N}$ is an amart, $\left\{\varphi\left(X_{n}\right), \mathscr{F}_{n}\right\}_{n \in N}$ is an $L^{1}$-bounded amart, provided $\left\{X_{n}^{-}\right\}_{n \in N}$ is $L^{1}$-bounded and $\left\{\left(X^{+}\right)^{2}\right\}_{r \in T}$ is uniformly integrable.

In the remainder of this section we use the examples from Section 3 to produce the examples that were promised at the end of Remark 5.4.

First, let $D=N$. Suppose that the assumption that $\left\{\varphi\left(X_{\tau}\right)\right\}_{\tau \in T}$ is uniformly integrable is replaced by the assumption that $\left\{\varphi\left(X_{n}\right)\right\}_{n \in N}$ is uniformly integrable and consider Example 3.2 together with the function $\varphi(x)=|x|^{p}, p>1$. Then, $\left\{X_{n}^{(p)}, \mathscr{F}_{n}^{(p)}\right\}_{n \in N}$ (with $p>1)$ is an $L^{1}$-bounded amart. Further, since $\varphi\left(X_{n}^{(p)}\right)=X_{n}^{(1)}$ it follows that $\left\{\varphi\left(X_{n}^{(p)}\right)\right\}_{n \in N}$ is uniformly integrable and that $\left\{\varphi\left(X_{n}^{(p)}\right), \mathscr{F}_{n}^{(p)}\right\}_{n \in N}$ fails to be an amart. The condition that $\left\{\varphi\left(X_{n}\right)\right\}_{n \in N}$ is uniformly integrable is thus not sufficient for Theorem 5.3 to hold in general (if $D=N$ ).

Next, consider a possible replacement with the assumption that $\left\{\varphi\left(X_{n}\right), \mathscr{F}_{n}\right\}_{n \in N}$ is an $L^{1}$-bounded semiamart (or, equivalently, that $\left\{\varphi\left(X_{\tau}\right)\right\}_{\tau \in T}$ is $L^{1}$-bounded) and apply Example 3.1 together with the function $\varphi(x)=|x|^{p}, p>1$. Then, $\left\{X_{n}^{(p)}, \mathscr{F}_{n}^{(p)}\right\}_{n \in N}(p>1)$ is an $L^{1}$-bounded amart and $\left\{\varphi\left(X_{n}^{(p)}\right), \mathscr{F}_{n}^{(p)}\right\}_{n \in N}$ is an $L^{1}$-bounded semiamart but not an amart.

Note that, since none of the conditions " $\left\{\varphi\left(X_{n}\right)\right\}_{n \in N}$ is uniformly integrable" and " $\left\{\varphi\left(X_{\mathrm{T}}\right)\right\}_{\tau \in \mathrm{T}}$ is $L$-bounded" imply each other (combine Example 3.2 with $1 / 2<p<1$ and Remark 3.4), both conditions had to be investigated.

Now, let $D=-N$. Suppose that the assumption that $\left\{\varphi\left(X_{n}\right), \mathscr{F}_{n}\right\}_{n \in-N}$ is a semiamart is weakened to the assumption that $\left\{\varphi\left(X_{n}\right)\right\}_{n \in-N}$ is uniformly integrable and consider Example 3.5 together with the function $\varphi(x)=|x|^{p}, p>1$. Then, $\left\{X_{n}^{(p)}, \mathscr{F}_{n}^{(p)}\right\}_{n \in-N}$, where $p>1$, is an amart, $\left\{\varphi\left(X_{n}^{(p)}\right)\right\}_{n \in-N}$ is uniformly integrable but $\left\{\varphi\left(X_{n}^{(p)}\right), \mathscr{F}_{n}^{(p)}\right\}_{n \in-N}$ is not an amart. 
6. On the Riesz decomposition when $D=-N$. The Riesz decomposition theorem for amarts has been proved in [7, Theorem 3.2] for the case $D=N$. The case $D=-N$ has been touched upon in $[\mathbf{1 0}$, p. 216] and this section contains some further facts.

Let $\left\{X_{n}, \mathscr{F}_{n}\right\}_{n \in-N}$ be an amart. It is well known from martingale theory that $\left\{E^{\mathscr{F}_{n}} X_{-1}, \mathscr{F}_{n}\right\}_{n \in-N}$ is a uniformly integrable martingale that converges a.s. and in $L^{1}$ as $n \rightarrow-\infty$. Also, from amart theory we know that $X_{n}$ converges a.s. and in $L^{1}$ to $X_{-\infty}$, say, as $n \rightarrow-\infty$.

Set, for $n \in-N$,

$$
\begin{gathered}
Y_{n}=E^{\mathscr{T}_{n}} X_{-1}+X_{-\infty}-E^{\mathscr{T}_{-\infty}} X_{-1}, \quad \text { and } \\
Z_{n}=X_{n}-Y_{n} .
\end{gathered}
$$

Now, since $X_{-\infty}$ and $E^{\mathscr{F}}-X_{-1}$ are $\mathscr{F}_{-\infty}$-measurable, i.e. $\mathscr{F}_{n}$-measurable for all $n \in-N$, $Y_{n}$ can also be written as

$$
Y_{n}=E^{\mathscr{F}_{n}}\left(X_{-1}+X_{-\infty}-E^{\mathscr{F}_{-\infty}} X_{-1}\right)
$$

It follows that $\left\{Y_{n}, \mathscr{F}_{n}\right\}_{n \in-N}$ is a martingale and that $\left\{Z_{n}, \mathscr{F}_{n}\right\}_{n \in-N}$ is an amart. Also, $\left\{Z_{\tau}\right\}_{r \in T}$ is uniformly integrable, since $D=-N$, and $Z_{n} \rightarrow 0$ a.s. and in $L^{1}$ as $n \rightarrow-\infty$. The details are omitted. The following theorem is thus obtained.

THEOREM 6.1. Suppose $D=-N$. Let $\left\{X_{n}, \mathscr{F}_{n}\right\}_{n \in-N}$ be an amart. Then $X_{n}$ can be written as $X_{n}=Y_{n}+Z_{n}$, where $\left\{Y_{n}, \mathscr{F}_{n}\right\}_{n \in-N}$ is a martingale and $\left\{Z_{n}, \mathscr{F}_{n}\right\}_{n \in-N}$ is an amart such that $Z_{n} \rightarrow 0$ a.s. and in $L^{1}$ as $n \rightarrow-\infty$ and (automatically) such that $\left\{Z_{4}\right\}_{\tau \in T}$ is uniformly integrable.

The proof of the uniqueness when $D=N$ is essentially based upon the fact that there are no non-trivial martingales that converge to 0 in $L^{1}$. When $D=-N$ this is no longer the case and the following example shows that there need not be uniqueness.

EXAmPLE 6.2. Consider Example 4.6 with $1<r<2$. The following decompositions are possible:

$$
\begin{aligned}
& X_{-n}=0+X_{-n} \\
& X_{-n}=\frac{1}{n} \sum_{k=1}^{n} \xi_{k}+\left(X_{-n}-\frac{1}{n} \sum_{k=1}^{n} \xi_{k}\right) .
\end{aligned}
$$

Since $\left\{X_{n}, \mathscr{F}_{n}\right\}_{n \in-N}$ is an amart that satisfies the properties of the potential part, the first decomposition is obvious. For the second one, set $Y_{-n}=n^{-1} . \sum_{k=1}^{n} \xi_{k}$. Then $\left\{Y_{n}, \mathscr{F}_{n}\right\}_{n \in-N}$ is a uniformly integrable martingale, $Y_{n} \rightarrow 0$ a.s. and in $L^{1}$ as $n \rightarrow-\infty$ and $X_{-n}-Y_{-n}$ is an amart that has the properties of the potential part as described in the theorem.

\section{REFERENCES}

1. D. G. Austin, G. A. Edgar, and A. Ionescu Tulcea, Pointwise convergence in terms of expectations, Z. Wahrsch. Verw. Gebiete 30 (1974), 17-26.

2. J. R. Baxter, Pointwise in terms of weak convergence, Proc. Amer. Math. Soc. 46 (1974), 395-398. 
3. A. Bellow, Stability properties of the class of asymptotic martingales, Bull. Amer. Math. Soc. 82 (1976), 338-340.

4. A. Bellow, Several stability properties of the class of asymptotic martingales, $Z$. Wahrsch. Verw. Gebiete 37 (1977), 275-290.

5. D. Blackwell and L. E. Dubins, A converse to the dominated convergence theorem, Illinois J. Math. 7 (1963), 508-514.

6. J. L. Doob, Stochastic processes (Wiley, 1953).

7. G. A. Edgar and L. Sucheston, Amarts: A class of asymptotic martingales. A. Discrete parameter, J. Multivariate Anal. 6 (1976), 193-221.

8. A. Gut, Moments of the maximum of normed partial sums of random variables with multidimensional indices, Z. Wahrsch. Verw. Gebiete 46 (1979), 205-220.

9. M. J. Klass, On stopping rules and the expected supremum of $S_{n} / a_{n}$ and $\left|S_{n}\right| / a_{n}, A n n$. Probab. 2 (1974), 889-905.

10. U. Krengel and L. Sucheston, On semiamarts, amarts and processes with finite value, Advances in Probability 4 (1978), 197-266.

11. M. Loève, Probability theory, 3rd edition (Van Nostrand, 1963).

12. J. F. Mertens, Théorie des processus stochastiques genéraux applications aux surmartingales, Z. Wahrsch. Verw. Gebiete 22 (1972), 45-68.

13. P. A. Meyer, Probabilités et Potentiel (Hermann, 1966).

14. J. Neveu, Discrete-parameter martingales (North-Holland, 1975).

15. W. Sudderth, A "Fatou equation" for randomly stopped variables, Ann. Math. Statist. 42 (1971), 2143-2146.

UPPSALA UNIVERSITY

DePaRTMENT OF Mathematics

S-752 38 UPPSALA

SWEDEN 